\title{
Calculations of Bell and Leinaas and Derbenev and Kondratenko for radiative electron polarization
}

\author{
D. P. Barber \\ Deutsches Elektronen-Synchrotron, Notkestrasse 85, D-2000 Hamburg 52, Federal Republic of Germany \\ S. R. Mane \\ Randall Laboratory of Physics, University of Michigan, Ann Arbor, Michigan 48109
}

(Received 28 April 1987)

\begin{abstract}
Derbenev and Kondratenko calculated the equilibrium degree of radiative electron polarization in 1973 (Ya. S. Derbenev and A. M. Kondratenko, Zh. Eksp. Teor. Fiz. 64, 1918 (1973) [Sov. Phys._JETP 37, 968 (1973)]), and more recently Bell and Leinaas did likewise for a more limited model, but following a different approach [J. S. Bell and J. M. Leinaas, Nucl. Phys. B 284, 488 (1987)]. They report a different resonance structure. In this paper the notations, formalisms, and viewpoints of the two sets of authors are compared, and the connection between their treatments is explained. The formula for the polarization, taking into account vertical fluctuations, is derived following the Derbenev-Kondratenko approach and is generalized to strong-focusing machines. It is also combined with the Derbenev-Kondratenko result into a unified formula.
\end{abstract}

\section{INTRODUCTION}

Derbenev and Kondratenko studied the problem of radiative electron polarization in the early 1970s, generalizing the work of previous authors, and gave a formula for the equilibrium degree of polarization in 1973, which is now called the Derbenev-Kondratenko formula. ${ }^{1}$ This formula described the effect, on the polarization, of electron energy loss due to photon emissions, but neglected vertical recoils by the electrons. The latter effect had been included in an earlier paper, ${ }^{2}$ but the treatment was incomplete. More recently, Bell and Leinaas, ${ }^{3}$ following a different approach, also calculated the equilibrium degree of radiative electron polarization, taking into account vertical electron recoils, and here again this work supersedes previous publications by these authors on the problem.

In this paper the notations, formalism, and viewpoints of the two sets of authors are compared, and the connection between their treatments is explained. Their results are also combined into a unified formula. For brevity the authors will be referred to as DK (Derbenev and Kondratenko) and BL (Bell and Leinaas), with an obvious notation. BL treat only a specific model, and so their results are valid in a more restricted domain of accelerator physics than those of DK. Their results also differ in a small energy region near a spin resonance. To quote BL (abstract of Ref. 3), "This analysis confirms the standard result for the polarization, except in the neighborhood of a narrow resonance." In Sec. VI we offer a more general treatment and explain how these points appear in Ref. 1. Unless otherwise stated, all references to DK will be to Ref. 1, not Ref. 2 .

\section{HAMILTONIAN}

Perhaps it would be simplest if the derivation of the equilibrium polarization were summarized afresh and comparisons with Refs. 1 and 3 were made along the way. The argument will largely follow that of Ref. 4. The unperturbed Hamiltonian is

$\mathscr{H}_{\mathrm{ext}}=\left[\left[\mathbf{p}-\frac{e}{c} \mathbf{A}_{\mathrm{ext}}\right]^{2} c^{2}+m^{2} c^{4}\right]^{1 / 2}+e \boldsymbol{\Phi}_{\mathrm{ext}}+\mathbf{\Omega}_{\mathrm{ext}} \cdot \mathbf{s}$

and the interaction Hamiltonian is

$$
\mathcal{H}_{\mathrm{int}}=e\left(\Phi_{\mathrm{rad}}-\boldsymbol{\beta} \cdot \mathbf{A}_{\mathrm{rad}}\right)+\mathbf{s} \cdot \boldsymbol{\Omega}_{\mathrm{rad}},
$$

where

$$
\begin{aligned}
\mathbf{\Omega}_{\mathrm{ext}, \mathrm{rad}}=-\frac{e}{m c}[ & {\left[a+\frac{1}{\gamma}\right] \mathbf{B}-\frac{a \gamma}{\gamma+1} \boldsymbol{\beta} \cdot \mathbf{B} \boldsymbol{\beta} } \\
& \left.-\left[a+\frac{1}{\gamma+1}\right] \boldsymbol{\beta} \times \mathbf{E}\right]_{\mathrm{ext}, \mathrm{rad}} .
\end{aligned}
$$

The notation is standard: $\mathbf{r}$ and $\mathbf{p}$ are the canonical electron position and momentum, respectively; $m, e$, and $s$ are the electron mass, charge, and spin; $\boldsymbol{\beta}$ is the electron velocity in units of $c$; and the subscripts ext and rad denote external (accelerator) and radiation fields. In Eq. (3) $a \equiv(g-2) / 2$; BL write $\kappa \equiv(g-2) / 2$. DK write $q_{0}$ and $q^{\prime}$, where $q_{0}=e / m c$ and $q^{\prime}=a e / m c$, and write $x=q^{\prime} / q_{0}=(g-2) / 2$.

It will be useful below to write $\Omega \cdot s$ in the form 


$$
\mathbf{\Omega} \cdot \mathbf{s}=-\frac{g e}{2 m c \gamma} \mathbf{s} \cdot \mathbf{B}^{\prime}+\omega_{T} \cdot \mathbf{s},
$$

where $\mathbf{B}^{\prime}$ is the rest-frame magnetic field and $\omega_{T}$ is the Thomas precession vector

$\omega_{T}=-\frac{e}{m c} \frac{\gamma}{\gamma+1} \boldsymbol{\beta} \times(\mathbf{E}+\boldsymbol{\beta} \times \mathbf{B})=-\frac{e}{m c(\gamma+1)} \boldsymbol{\beta} \times \mathbf{E}^{\prime}$,

where $\mathbf{E}^{\prime}$ is the rest-frame electric field. The use of this form for the Hamiltonian will simplify comparison with the work of BL.

DK use Eqs. (1) and (2), which pertain to the laboratory (accelerator) frame. BL start from the Dirac Hamiltonian, and as with DK, they introduce a coupling to the anomalous magnetic moment, following a standard procedure. They also make a canonical transformation to the comoving (electron) frame, and in this frame they make a Foldy-Wouthuysen transformation to first order in $\hbar$ to bring the Hamiltonian to semiclassical form. See Eqs. (4)-(17) of Ref. 3.

\section{DIAGONALIZATION}

Now BL restrict attention to perfectly aligned weakfocusing storage rings, where the accelerator fields are constant, i.e., independent of arc length, around the ring. The field is vertical on the planar closed orbit and has a gradient index $n=-(R / B) \partial B / \partial r$, where $R$ is the machine radius, $B$ is the magnetic field strength, and $r$ is the radial displacement from the closed orbit. Their term "classical particle orbit" denotes only the closed orbit (a circle), not the betatron oscillations. When DK refer to the "particle trajectory," they mean an arbitrary trajectory, not just the closed orbit. This use of words will have some bearing on their respective statements concerning the diagonalization of the spin-dependent part of the Hamiltonian, which will now be described.

BL divide the Hamiltonian into unperturbed and interaction terms slightly differently from Eqs. (1) and (2). In Sec. 3 of Ref. 3, they write

$$
H_{\text {spin }}=\frac{1}{2} \hbar \omega \cdot \sigma
$$

where $\sigma=(2 / \hbar)$ s and $\omega$ contains both external and radiation fields. These are all operators in the comoving, not the accelerator, frame. They divide $\omega$ into a classical part $\omega_{1}$ and a quantum part $\delta \omega$. The classical part pertains to motion on the classical particle orbit, i.e., the closed orbit, which is a horizontal circle, and the quantum part contains everything else, including betatron oscillations. Thus, $\omega_{i} \sigma \propto \sigma \cdot \hat{z}$ and so they choose $\hat{\mathbf{z}}$ as their quantization axis; it diagonalizes their unperturbed Hamiltonian.

Let us now look at how DK perform the diagonalization. The Heisenberg equation of spin motion is the Thomas-Bargmann-Michel-Telegdi (Thomas-BMT) equation

$$
\frac{d \mathbf{s}}{d t}=\frac{1}{i \hbar}\left[\mathbf{s}, \mathcal{H}_{\mathrm{ext}}\right]=\frac{1}{i \hbar}\left[\mathbf{s}, \mathbf{s} \cdot \mathbf{\Omega}_{\mathrm{ext}}\right]=\mathbf{\Omega}_{\mathrm{ext}} \times \mathbf{s},
$$

and note that $\boldsymbol{\Omega}_{\text {ext }}=\boldsymbol{\Omega}_{\text {ext }}(\mathbf{r}, \mathbf{p})$; it depends on the orbital trajectory. Diagonalizing the spin-dependent part of the Hamiltonian basically means finding the right quantization axis; let us call it $\hat{\mathbf{n}}$. Since $\boldsymbol{\Omega}_{\text {ext }}$ depends on the orbital trajectory, which is not always the closed orbit, DK pointed out that $\hat{\mathbf{n}}$ must depend on the orbit, too. The Heisenberg equation of motion for the operator $\mathbf{s} \cdot \hat{\mathbf{n}}$, which measures the spin projection along $\widehat{\mathbf{n}}$, is $^{4}$

$$
\frac{d}{d t}(\mathbf{s} \cdot \hat{\mathbf{n}})=\frac{1}{i \hbar}\left[\mathbf{s} \cdot \hat{\mathbf{n}}, \mathcal{H}_{\mathrm{ext}}\right]+\frac{\partial}{\partial t}(\mathbf{s} \cdot \hat{\mathbf{n}}) .
$$

Following the article by Yokoya, ${ }^{5}$ in which some aspects of the DK work are clarified, $\hat{\mathbf{n}}$ is defined to be the explicitly time-independent solution of $\mathrm{Eq}$. (7) on a given orbital trajectory: $\widehat{\mathbf{n}}=\widehat{\mathbf{n}}(\mathbf{r}, \mathbf{p})$ only, i.e., $\widehat{\mathbf{n}} \neq \widehat{\mathbf{n}}(\mathbf{r}, \mathbf{p}, t)$. Actually, since $\hat{\mathbf{n}}$ is a classical vector, not a quantum operator, $d \hat{\mathbf{n}} / d t=\left\langle\boldsymbol{\Omega}_{\text {ext }}\right\rangle \times \hat{\mathbf{n}}$, where $\left\langle\boldsymbol{\Omega}_{\text {ext }}\right\rangle$ is the expectation value of $\Omega_{\text {ext }}$ over the electron state. By the properties of $\mathbf{s}$ and $\hat{\mathbf{n}}, \partial(\mathbf{s} \cdot \hat{\mathbf{n}}) / \partial t=0$, and also

$$
\frac{d}{d t}(\mathbf{s} \cdot \hat{\mathbf{n}})=\left(\boldsymbol{\Omega}_{\mathrm{ext}} \times \mathbf{s}\right) \cdot \hat{\mathbf{n}}+\mathbf{s} \cdot\left(\left\langle\boldsymbol{\Omega}_{\mathrm{ext}}\right\rangle \times \hat{\mathbf{n}}\right) \simeq 0,
$$

the symbol $\simeq 0$ being used because the right-hand side actually vanishes only to the leading order in $\hbar$. Thus, in Eq. (8), the commutator [s. $\left.\hat{\mathbf{n}}, \mathscr{H}_{\text {ext }}\right]$ vanishes, to the leading order in $\hbar$, and we have diagonalized the unperturbed Hamiltonian, to that order.

Note that in a uniform magnetic field, $\hat{\mathbf{n}}(\mathrm{DK})$ is the direction of the field, and so $\hat{\mathbf{n}}=\hat{\mathbf{z}}$ on the closed orbit of the $\mathrm{BL}$ model. So $\mathrm{DK}$ and $\mathrm{BL}$ are not using grossly different quantization axes.

\section{PERTURBATIONS}

Let us now consider the perturbations. DK do not describe the calculation of the orbital equilibrium explicitly, but they assume it to be determined by the standard ingredients of radiation damping and stochastic excitation. BL basically do the same, but they do it explicitly, using a Langevin equation to calculate the equilibrium emittance $\left\langle z^{2}\right\rangle$ of the vertical betatron oscillations. They use the terms "quantum fluctuations" from the "classical orbit" instead of "stochastic excitations" from the closed orbit. Note that both sets of authors take into account the fact that the energy loss in synchrotron radiation is compensated, and that the whole thing results in damping and stochastic excitations and/or quantum fluctuations; the original work of Sokolov and Ternov ${ }^{6}$ (solution of the Dirac equation in a uniform, static, vertical magnetic field, with second-quantized radiation field) did not.

At this point the main difference between Refs. 1 and 3 appears; to calculate the vertical emittance $\mathrm{BL}$ take into account the vertical recoil of the electron when emitting a photon. For electrons moving mainly horizontally, such terms are very small, and are neglected by DK in Ref. 1. They are included in Ref. 2, but the treatment is incomplete. For this reason BL obtain a different result from DK, but in Sec. VI the results of Refs. 1-3 will be unified into a general formula.

Considering now the spin-dependent perturbations, $\mathbf{s} \cdot \hat{\mathbf{n}}$ or $\boldsymbol{\sigma} \cdot \hat{\mathbf{z}}$ is no longer a constant of the motion in the 
presence of radiation. In the quantum theory (spin $\frac{1}{2}$ ), $\mathbf{s} \cdot \hat{\mathbf{n}}$ can have one of only two values, and the radiation causes transitions between them,

$$
\begin{aligned}
\frac{d}{d t}(\mathbf{s} \cdot \hat{\mathbf{n}}) & =\left\{\mathbf{s} \cdot \hat{\mathbf{n}}, \mathscr{H}_{\mathrm{int}}\right\} \\
& =\left\{\mathbf{s}, \mathcal{H}_{\mathrm{int}}\right\} \cdot \hat{\mathbf{n}}+\mathbf{s} \cdot\left\{\hat{\mathbf{n}}, \mathscr{H}_{\mathrm{int}}\right\} \\
& \simeq\left(\boldsymbol{\Omega}_{\mathrm{rad}} \times \mathbf{s}\right) \cdot \hat{\mathbf{n}}+\mathbf{s} \cdot\left\{\hat{\mathbf{n}}, e\left(\Phi_{\mathrm{rad}}-\boldsymbol{\beta} \cdot \mathbf{A}_{\mathrm{rad}}\right)\right\} .
\end{aligned}
$$

Poisson brackets have been used instead of quantum commutators because Eq. (10) is taken from DK, and they used Poisson brackets. Note that there are two contributions to the fluctuations in $\mathbf{s} \cdot \hat{\mathbf{n}}$, and they must be added in the spin-flip matrix elements, not in the transition rates. DK misunderstood this point in their early work in Ref. 2. In Eq. (4.13) they calculated a "depolarization time" which had to be combined with a polarization time in order to arrive at the equilibrium polarization. This means that they were adding the terms in the transition rates, which is why their treatment there was incomplete, as stated above. They corrected themselves later on in Ref. 1. DK call the first term the "direct interaction" and the second "spin-orbit coupling." BL call the first term the "basic polarization mechanism" and the second the "vertical fluctuation effect." To quote them, "With the spin-orbit coupling taken into account, the radiation can thus act on the polarization not only directly, but also via the trajectory, perturbing the quantization axis" (DK, Ref. 1) and, ". . . we treat the vertical fluctuation effect coherently with the basic polarization mechanism. .." (BL, Ref. 3).

Equation (10) is in the DK form, which is applicable to arbitrary electron trajectories. The BL calculation of quantum fluctuations, etc., is only valid in a small region near the closed orbit (where $\hat{\mathbf{n}}=\hat{\mathbf{z}}$ ). We shall see later that because of this they obtain only first-order spin resonances in their calculation. Both DK and BL combine the above mechanisms of spin flip to obtain effective interaction Hamiltonians before calculating the matrix elements and transition probabilities per unit time. In other words, they include the kinetics of orbital motion, which is not present in $H_{\text {int }}$ of Eq. (2), into the effective interaction Hamiltonian. They come up with different functions, obviously, but the connection between them will now be established.

\section{EQUILIBRIUM POLARIZATION}

BL take into account vertical recoils due to photon emissions, and write

$$
m \ddot{z}=e\left(E_{q z}^{\prime}+\delta E_{c z}^{\prime}\right),
$$

where the primes denote evaluation in the comoving frame, $\delta E_{c}^{\prime}$ is the field seen due to motion off the closed orbit (vertical betatron oscillations), and $E_{q}^{\prime}$ is the quantum part of the radiation electric field, including radiation damping. BL write $E_{q z}^{\prime}=E_{f z}^{\prime}+E_{s z}^{\prime}$, where $E_{f z}^{\prime}$ is the free radiation field, and

$$
E_{s z}^{\prime}=\frac{2}{3} \frac{e^{2}}{c^{3}}\left(\dddot{z}-\gamma^{4} \omega_{0}^{2} \dot{z}\right)
$$

describes the radiation damping. They put

$$
\delta E_{c z}^{\prime}=-\gamma^{2} \omega_{0}^{2} Q^{2}(m / e) z,
$$

i.e., they assume that the vertical betatron oscillations are exactly harmonic. Here $Q$ is the vertical betatron tune, $Q=\sqrt{n}$, where $n=-(R / B) \partial B / \partial r$ is the field gradient index. They call their effective interaction Hamiltonian $\hbar \delta \omega \cdot \sigma / 2$ and calculate

$$
\begin{aligned}
\delta \omega_{ \pm} \equiv \delta \omega \cdot(\hat{\mathbf{x}} \pm i \hat{\mathbf{y}}) & =-\frac{e}{2 m c}\left[g \delta B_{ \pm}^{\prime}+2 \beta \frac{m}{e} \ddot{z}\right] \\
& =-\frac{e}{2 m c}\left[g B_{f \pm}^{\prime}+\left(2+f_{ \pm}\right) E_{f z}^{\prime}\right],
\end{aligned}
$$

where $E_{f}^{\prime}$ and $B_{f}^{\prime}$ are the free radiation electric and magnetic fields, respectively, and

$$
f_{ \pm}=\frac{(g-2) \gamma^{2} Q^{2}}{\gamma^{2} Q^{2}-\gamma^{2} v^{2} \pm i \Delta \gamma \nu / \omega_{0}}
$$

and

$$
\Delta=\frac{2}{3} \frac{e^{2}}{m c^{3}}\left(\gamma^{4} \omega_{0}^{2}+\gamma^{2} \omega_{0}^{2} v^{2}\right),
$$

where $\omega_{0}=\beta c / R, Q$ is the vertical betatron tune, and $v=\gamma(g-2) / 2$ is the spin tune. They neglect the term in $\Delta$, and write

$$
f=\frac{(g-2) Q^{2}}{Q^{2}-v^{2}},
$$

and from this they obtain their formula for the equilibrium degree of polarization,

$$
P_{\text {eq }}=\frac{8}{5 \sqrt{3}} \frac{1-\frac{f}{6}}{1-\frac{f}{18}+\frac{13}{360} f^{2}} .
$$

This formula has a resonance at $v=Q=\sqrt{n}$, or $\gamma=2 \sqrt{n} /(g-2)$. This agrees with the previously known result for the locations of resonances

$$
\nu=k_{0}+k_{x} Q_{x}+k_{z} Q_{z}+k_{s} Q_{s},
$$

where $Q_{x}, Q_{z}$, and $Q_{s}$ are the orbital tunes and $k_{0}, \ldots$ are integers, including zero. $\mathrm{BL}$ report that as $\gamma$ moves through the resonance from below, $P_{\text {eq }}$ drops from $8 /(5 \sqrt{3}) \simeq 0.924$ to -0.169 , but then increases to 0.992 before falling again to 0.924 . This can be checked by a simple computer calculation. It is found that the extremum values for $P_{\text {eq }}$ do not depend on the value of $n$, but the width of the resonance does, becoming larger as $n$ increases.

Let us now consider the DK work. They call their effective interaction Hamiltonian $\omega \cdot \mathbf{s}$, defined such that

$$
\frac{d}{d t}(\mathbf{s} \cdot \hat{\mathbf{n}})=\left\{\mathbf{s} \cdot \hat{\mathbf{n}}, \mathcal{H}_{\text {int }}^{\text {eff }}\right\}=\{\mathbf{s} \cdot \hat{\mathbf{n}}, \boldsymbol{\omega} \cdot \mathbf{s}\}=(\boldsymbol{\omega} \times \mathbf{s}) \cdot \hat{\mathbf{n}},
$$

and using Eq. (10), they derive 


$$
\boldsymbol{\omega}=\boldsymbol{\Omega}_{\mathrm{rad}}-\hat{\mathbf{n}} \times\left(\mathbf{F} \cdot \frac{\partial}{\partial \mathbf{p}}\right) \widehat{\mathbf{n}}
$$

where $\mathbf{F}=e\left(\mathbf{E}_{\mathrm{rad}}+\boldsymbol{B} \times \mathbf{B}_{\mathrm{rad}}\right)$. The fields are of course in the laboratory frame now. DK neglect vertical recoil, and assume that the only effect of photon emissions is energy loss (but see below), in which case $\partial / \partial \mathbf{p} \simeq(\beta / m c) \partial / \partial \gamma$, so that

$$
\boldsymbol{\omega} \simeq \boldsymbol{\Omega}_{\mathrm{rad}}-\frac{e}{m c} \boldsymbol{\beta} \cdot \mathbf{E}_{\mathrm{rad}} \hat{\mathbf{n}} \times \frac{\partial \widehat{\mathbf{n}}}{\partial \gamma} .
$$

From this they obtain their polarization formula

$$
P_{\text {eq }}=\frac{8}{5 \sqrt{3}} \frac{\left\langle\frac{1}{|\rho|^{3}} \hat{\mathbf{b}} \cdot\left(\hat{\mathbf{n}}-\gamma \frac{\partial \hat{\mathbf{n}}}{\partial \gamma}\right)\right\rangle}{\left.\left\langle\left.\frac{1}{|\rho|^{3}}\left|1-\frac{2}{9}(\hat{\mathbf{n}} \cdot \hat{\mathbf{v}})^{2}+\frac{11}{18}\right| \gamma \frac{\partial \hat{\mathbf{n}}}{\partial \gamma}\right|^{2}\right)\right\rangle},
$$

with resonances as in Eq. (18). Here $\hat{\mathbf{b}}=\mathbf{v} \times \dot{\mathbf{v}} /|\mathbf{v} \times \dot{\mathbf{v}}|$ and $\rho$ is the local bending radius of the trajectory. Now, for the weak-focusing model of BL, it can be shown that $\gamma(\partial \hat{\mathbf{n}} / \partial \gamma)=0$, and so the above formula cannot reproduce the $B L$ result.

DK neglect the vertical quantum fluctuations at the beginning of Sec. 6 of Ref. 1, where they say (using our mathematical notation), "In the case of motion in inhomogeneous fields, the gradients of $\mathbf{n}$ in the longitudinal and transverse directions are generally speaking of the same order." Recognizing that $\mathbf{F}_{v} \sim \gamma \mathbf{F}_{t r}$, we obtain the following expression for $\omega$ :

$$
\boldsymbol{\omega} \approx-\frac{e}{\gamma m c} \mathbf{B}_{v}-\frac{e}{\gamma^{2} m c} \mathbf{B}_{t r}-\frac{e}{m c} \boldsymbol{\beta} \cdot \mathbf{E} \hat{\mathbf{n}} \times \frac{\partial \hat{\mathbf{n}}}{\partial \gamma} .
$$

The first sentence states that

$$
\left|\gamma \frac{\partial \widehat{\mathbf{n}}}{\partial \gamma}\right| \simeq\left|\frac{\partial \widehat{\mathbf{n}}}{\partial \beta_{z}}\right|
$$

In the second sentence, $\mathbf{F} \equiv e(\mathbf{E}+\boldsymbol{B} \times \mathbf{B}) \equiv \mathbf{F}_{v}+\mathbf{F}_{t r}$. Now

$$
\omega_{ \pm} \equiv \omega \cdot(\hat{\mathbf{x}} \pm i \hat{\mathbf{y}}) \simeq-\frac{e}{2 m c \gamma}\left[g B_{f \pm}^{\prime}+2(\hat{\mathbf{x}} \pm i \hat{\mathbf{y}}) \cdot\left(\hat{\mathbf{y}} \times \mathbf{E}_{f}^{\prime}\right)+\frac{2}{\gamma} E_{f z}^{\prime}(\hat{\mathbf{x}} \pm i \hat{\mathbf{y}}) \cdot\left(\widehat{\mathbf{z}} \times \frac{\partial \hat{\mathbf{n}}}{\partial \beta_{z}}\right]\right]
$$

where the approximations $\beta \simeq \widehat{\mathbf{y}}$ and $\hat{\mathbf{n}} \simeq \hat{\mathbf{z}}$ have been used. The above result simplifies to

$\omega_{ \pm} \simeq-\frac{e}{2 m c \gamma}\left[g B_{f \pm}^{\prime}+2 E_{f z}^{\prime}+\frac{2}{\gamma} E_{f z}^{\prime}(-\hat{\mathbf{y}} \pm i \widehat{\mathbf{x}}) \cdot \frac{\partial \widehat{\mathbf{n}}}{\partial \beta_{z}}\right]$.

$$
\begin{aligned}
\boldsymbol{\omega} & =\mathbf{\Omega}-\hat{\mathbf{n}} \times\left(\mathbf{F} \cdot \frac{\partial}{\partial \mathbf{p}}\right) \hat{\mathbf{n}} \\
& \simeq \mathbf{\Omega}-\hat{\mathbf{n}} \times\left(\frac{F_{y}}{m c} \frac{\partial}{\partial \gamma}\right) \widehat{\mathbf{n}}-\widehat{\mathbf{n}} \times\left(\frac{F_{x}}{\gamma m c} \frac{\partial}{\partial \beta_{z}}\right) \widehat{\mathbf{n}} \\
& =\mathbf{\Omega}-\frac{F_{y}}{\gamma m c} \hat{\mathbf{n}} \times \gamma \frac{\partial \hat{\mathbf{n}}}{\partial \gamma}-\frac{F_{z}}{\gamma m c} \hat{\mathbf{n}} \times \frac{\partial \hat{\mathbf{n}}}{\partial \beta_{z}} .
\end{aligned}
$$

Since $F_{y} \simeq \gamma F_{z} \quad$ and $\quad \gamma \gg 1$ and $|\gamma(\partial \hat{\mathbf{n}} / \partial \gamma)|$ $\simeq\left|\partial \hat{\mathbf{n}} / \partial \beta_{z}\right|$, the last term is neglected, which yields the DK expression for $\omega$. It so happens that in a perfect planar storage ring, $\gamma(\partial \widehat{\mathbf{n}} / \partial \gamma)=0$ (see Appendix), so that the above approximation breaks down.

\section{RELATIONSHIP OF FORMALISMS}

However, the DK formalism can incorporate the effects of vertical recoil. Let us derive the polarization, taking into account vertical recoils, following the DK approach. To avoid cluttering the formula with inessential material, let us ignore $\partial \widehat{\mathbf{n}} / \partial \gamma$, which vanishes anyway for the model in question. Then

$$
\frac{\partial}{\partial \mathbf{p}} \simeq \frac{1}{\gamma m c} \widehat{\mathbf{z}} \frac{\partial}{\partial \beta_{z}}
$$

and so

$$
\boldsymbol{\omega} \simeq \boldsymbol{\Omega}_{\mathrm{rad}}-\frac{e}{\gamma m c} \hat{\mathbf{z}} \cdot\left(\mathbf{E}_{\mathrm{rad}}+\boldsymbol{\beta} \times \mathbf{B}_{\mathrm{rad}}\right) \hat{\mathbf{n}} \times \frac{\partial \hat{\mathbf{n}}}{\partial \beta_{z}} .
$$

Now note that the photon is emitted almost longitudinally, so that

$$
\widehat{\mathbf{z}} \cdot\left(\mathbf{E}_{\mathrm{rad}}+\boldsymbol{\beta} \times \mathbf{B}_{\mathrm{rad}}\right) \simeq \widehat{\mathbf{z}} \cdot \frac{\mathbf{E}_{\mathrm{rad}}^{\prime}}{\gamma}=\frac{1}{\gamma} E_{f z}^{\prime},
$$

in $\mathrm{BL}$ notation. Recall that $E_{f}^{\prime}$ is the rest-frame-free radiation electric field. Also, let us rewrite $\boldsymbol{\Omega}_{\text {rad }}$ using Eqs. (4) and (5),

$$
\Omega=-\frac{g e}{2 m c \gamma} \mathbf{B}^{\prime}+\omega_{T}=-\frac{g e}{2 m c \gamma} \mathbf{B}^{\prime}-\frac{e}{m c(\gamma+1)} \boldsymbol{\beta} \times \mathbf{E}^{\prime}
$$

and so

$$
\mathbf{\Omega}_{\mathrm{rad}} \simeq-\frac{e}{2 m c \gamma}\left(g \mathbf{B}_{f}^{\prime}+2 \boldsymbol{\beta} \times \mathbf{E}_{f}^{\prime}\right) .
$$

Hence,
There is an overall factor of $\gamma^{-1}$ from time dilation; otherwise, this agrees with the BL expression $\delta \omega_{ \pm}$provided $(2 / \gamma)(-\hat{\mathbf{y}} \pm i \widehat{\mathbf{x}}) . \quad \partial \hat{\mathbf{n}} / \partial \beta_{z}$ equals $f$ of Eq. (16).

We therefore evaluate $\partial \hat{\mathbf{n}} / \partial \beta_{z}$ to first order in the vertical betatron oscillations, followed by substitution into Eq. (32). To do this we first solve for $\hat{\mathbf{n}}$ to first or- 
der in $z$. For a weak-focusing machine, the vertical $\beta$ function is constant and so, using the normalization of Ref. 4,

$$
z=a_{z} \sqrt{\beta / 2} \exp (i Q \theta)+\text { c.c. },
$$

where $\theta$ is the machine azimuth, $Q=\sqrt{n}$ is the vertical betatron tune, $\beta=R / Q, a_{z}$ is a constant, and the weakfocusing quadrupole strength is $k=n / R=Q^{2} / R$. Clearly there is a problem of confusion with other meanings for $\beta, k$, etc., but there is no simple solution to this, so the reader is simply advised to be careful. Now we must solve Eq. (7) for $\widehat{\mathbf{n}}$. It is more convenient to use $\theta$ as the independent variable, and so we write

$$
\frac{d \hat{\mathbf{n}}}{d \theta}=\left(\mathbf{\Omega}_{0}+\mathbf{\Omega}_{\beta}\right) \times \hat{\mathbf{n}},
$$

where $\boldsymbol{\Omega}_{0}=(1+\gamma a) \hat{\mathbf{z}}$ is the spin precession vector on the closed orbit and

$$
\mathbf{\Omega}_{\beta}=-\frac{e}{m c \omega_{0}}\left[\left(a+\frac{1}{\gamma}\right) \mathbf{B}-\frac{a \gamma}{\gamma+1} \boldsymbol{\beta} \cdot \mathbf{B} \boldsymbol{\beta}\right]-\mathbf{\Omega}_{0},
$$

where $a=(g-2) / 2$. Using Eq. (33),

$$
\begin{aligned}
\mathbf{\Omega}_{\beta}(\theta) & \simeq(1+\gamma a) k z \hat{\mathbf{x}}+\frac{\gamma a}{R} \frac{d z}{d \theta} \hat{\mathbf{y}} \\
& =a_{z}\left[(1+\gamma a) k\left[\frac{\beta}{2}\right]^{1 / 2} \hat{\mathbf{x}}+i \frac{\gamma a}{\sqrt{2 \beta}} \hat{\mathbf{y}}\right] e^{i Q \theta}+\text { c.c. },
\end{aligned}
$$

whence

$$
\Omega_{\beta \pm}=a_{z}\left[(1+\gamma a) k\left[\frac{\beta}{2}\right]^{1 / 2} \mp \frac{\gamma a}{\sqrt{2 \beta}}\right] e^{i(Q \mp v) \theta}+\text { c.c. }
$$

This is Eq. (4.11) of Ref. 2, in different notation. Then, to first order in $\Omega_{\beta}$, it can be shown that ${ }^{4,7}$

$$
\begin{aligned}
\hat{n}(\theta) \simeq \hat{\mathbf{z}}-i \frac{\hat{\mathbf{x}}-i \hat{\mathbf{y}}}{2} \int_{-\infty}^{\theta} \Omega_{\beta+}\left(\theta^{\prime}\right) d \theta^{\prime}+i \frac{\hat{\mathbf{x}}+i \hat{\mathbf{y}}}{2} \int_{-\infty}^{\theta} \Omega_{\beta-}\left(\theta^{\prime}\right) d \theta^{\prime} \\
=\hat{\mathbf{z}}+\left\{-\frac{(\hat{\mathbf{x}}-i \hat{\mathbf{y}})}{2} a_{z}\left[(1+\gamma a) k\left[\frac{\beta}{2}\right)^{1 / 2}-\frac{\gamma a}{\sqrt{2 \beta}}\right] \frac{e^{i Q \theta}}{Q-v}\right. \\
\left.+\frac{(\hat{\mathbf{x}}+i \hat{\mathbf{y}})}{2} a_{z}\left[(1+\gamma a) k\left[\frac{\beta}{2}\right)^{1 / 2}+\frac{\gamma a}{\sqrt{2 \beta}}\right] \frac{e^{i Q \theta}}{Q+v}+\text { c.c. }\right\} .
\end{aligned}
$$

Some details on the partial differentiation $\partial / \partial \beta_{z}$ are given in the Appendix. The result is simply to replace $a_{z}$ by $i \sqrt{\beta / 2} \exp (-i Q \theta)$, whence

$$
\begin{aligned}
\frac{\partial \hat{\mathbf{n}}}{\partial \beta_{z}}= & -\frac{i}{4}(\hat{\mathbf{x}}-i \hat{\mathbf{y}})[(1+\gamma a) k \beta-\gamma a] \frac{1}{Q-v} \\
& +\frac{i}{4}(\hat{\mathbf{x}}+i \hat{\mathbf{y}})[(1+\gamma a) k \beta+\gamma a] \frac{1}{Q+v}+\text { c.c. } \\
= & -\hat{\mathbf{y}}\left[(1+\gamma a) \frac{Q^{2}}{Q^{2}-v^{2}}-\frac{v^{2}}{Q^{2}-v^{2}}\right] \\
= & -\hat{\mathbf{y}}\left[1+\frac{v Q^{2}}{Q^{2}-v^{2}}\right]
\end{aligned}
$$

because $v=\gamma a$. Thus

$$
\frac{2}{\gamma}(-\hat{\mathbf{y}} \pm i \widehat{\mathbf{x}}) \cdot \frac{\partial \hat{\mathbf{n}}}{\partial \beta_{z}} \simeq \frac{2}{\gamma}\left[1-\frac{v Q^{2}}{v^{2}-Q^{2}}\right]=\frac{2}{\gamma}+\frac{(g-2) Q^{2}}{Q^{2}-v^{2}}
$$

This is exactly the form which appears in Eq. (4.13) of Ref. 2, and so our calculation for the quadratic terms agrees with theirs [see Eq. (41) below]. The BL calculation yields the final term $(g-2) Q^{2} /\left(Q^{2}-v^{2}\right)$, which is the $f$ of Eq. (16). The additional term in Eq. (40), however, is finite at a spin resonance, whereas $f$ in Eq. (16) goes to infinity, and so near to the resonance we may consider the above results to be in agreement.

One of us (S.R.M.) has rederived the polarization, but using Eq. (40), and has generalized it to strong-focusing storage rings. The basis $\{\hat{\mathbf{x}}, \hat{\mathbf{y}}, \hat{\mathbf{z}}\}$ is reinterpreted as meaning $\hat{\mathbf{y}}=\hat{\mathbf{v}}, \hat{\mathbf{x}}=-\dot{\mathbf{v}} /|\dot{\mathbf{v}}|$, and $\hat{\mathbf{z}}=\hat{\mathbf{x}} \times \hat{\mathbf{y}} \equiv \hat{\mathbf{b}}$ is the direction of the field, not necessarily vertical. Next we define $\mathbf{f} \equiv-(2 / \gamma) \partial \hat{\mathbf{n}} / \partial \beta_{b}$, a vector, and it does not in general point along $\hat{\mathbf{v}}$. Note that horizontal betatron and synchrotron oscillations will also now contribute to f, in general. Then

$$
P_{\mathrm{eq}}=\frac{8}{5 \sqrt{3}} \frac{\left\langle\frac{1}{|\rho|^{3}}\left(\hat{\mathbf{n}} \cdot \hat{\mathbf{b}}-\frac{1}{6} \hat{\mathbf{v}} \cdot \mathbf{f}\right)\right\rangle}{\left\langle\frac{1}{|\rho|^{3}}\left(1-\frac{2}{9}(\hat{\mathbf{n}} \cdot \hat{\mathbf{v}})^{2}-\frac{1}{18} \frac{\dot{\mathbf{v}}}{|\dot{\mathbf{v}}|} \cdot(\hat{\mathbf{n}} \times \mathbf{f})+\frac{13}{360}|\mathbf{f}|^{2}\right]\right\rangle},
$$


where $\rho$ is the local bending radius of the trajectory, and the angular brackets denote an average over the distribution of particle orbits and the ring azimuth. DK did in fact almost obtain this result, for a perfect planar weak-focusing storage ring, as implied by Eq. (4.13) of Ref. 2. Because they added the spin-flip mechanisms of Eq. (10) only in the transition rates, not in the matrix elements, Eq. (4.13) of Ref. 2 contains only the quadratic, not the linear, terms in $f$.

The derivative $\partial \hat{\mathbf{n}} / \partial \gamma$, which describes the effects of energy loss on the spin, has been neglected above. Putting it in, it is found that no cross terms between $\partial \hat{\mathbf{n}} / \partial \gamma$ and $\partial \hat{\mathbf{n}} / \partial \beta_{b}$ appear, and so the formula which describes the combined set of resonances is

$$
P_{\text {eq }}=\frac{8}{5 \sqrt{3}} \frac{\left.\left.\left\langle\frac{1}{|\rho|^{3}}\right| \hat{\mathbf{n}} \cdot \hat{\mathbf{b}}-\gamma \frac{\partial \hat{\mathbf{n}}}{\partial \gamma} \cdot \hat{\mathbf{b}}+\frac{1}{3 \gamma} \frac{\partial \hat{\mathbf{n}}}{\partial \beta_{b}} \cdot \hat{\mathbf{v}}\right)\right\rangle}{\left.\left\langle\frac{1}{|\rho|^{3}}\left[1-\frac{2}{9}(\hat{\mathbf{n}} \cdot \hat{\mathbf{v}})^{2}+\frac{11}{18}\left|\gamma \frac{\partial \hat{\mathbf{n}}}{\partial \gamma}\right|^{2}+\frac{1}{9 \gamma} \frac{\dot{\mathbf{v}}}{|\dot{\mathbf{v}}|} \cdot \mid \hat{\mathbf{n}} \times \frac{\partial \hat{\mathbf{n}}}{\partial \beta_{b}}\right]+\frac{13}{90 \gamma^{2}}\left|\frac{\partial \widehat{\mathbf{n}}}{\partial \beta_{b}}\right|^{2}\right]\right\rangle} .
$$

\section{TRANSITION PROBABILITIES}

Both DK and BL use "integrals of free field correlation functions" [Eq. (46), Ref. 3] to calculate the spin-flip transition probabilities per unit time. Let us start with the BL integrals. Using Eq. (46) of Ref. 3,

$\Gamma_{ \pm}=\frac{1}{4} \int_{-\infty}^{\infty} d \tau \exp \left(\mp i \omega_{1} \tau\right)\left\langle\delta \omega_{ \pm}(\tau / 2) \delta \omega_{\mp}(-\tau / 2)\right\rangle$

where the subscripts \pm pertain to spin flips from $\mp \hat{\mathbf{z}}$ to $\pm \hat{\mathbf{z}}$, respectively. The quantity $\omega_{1}$ is the spin precession angular frequency on the classical orbit (closed orbit) [see Eqs. (2), (3), and (21) of Ref. 3],

$$
\omega_{1}=\gamma \omega_{0} \gamma \frac{g-2}{2}, \omega_{0}=\frac{\beta c}{R} .
$$

This agrees with the DK expression $\Omega=\omega_{0} \gamma(g-2) / 2$ for this model, the extra factor of $\gamma$ in Eq. (44) coming from time dilation.

DK write almost the same expressions, in fact. Instead of spin flips from only $\pm \hat{\mathbf{z}}$ to $\mp \hat{\mathbf{z}}$, they consider spin flips from $\pm \hat{\mathbf{n}}$ to $\mp \hat{\mathbf{n}}$. DK call their effective interaction Hamiltonian $\omega \cdot \mathbf{s}$, whereas BL call theirs $(\hbar / 2) \delta \omega \cdot \sigma$. Thus, instead of using $\delta \omega_{ \pm}=\delta \omega_{x} \pm i \delta \omega_{y}$, they use $\omega \cdot \boldsymbol{\eta} \equiv \omega \cdot\left(\boldsymbol{\eta}_{1}+i \boldsymbol{\eta}_{2}\right)$ and $\omega \cdot \boldsymbol{\eta}^{*}$, where $\boldsymbol{\eta}_{1}$ and $\boldsymbol{\eta}_{2}$ are unit vectors orthogonal to $\hat{\mathbf{n}}$ and to each other, and are both solutions of the Thomas-BMT equation on a given orbital trajectory (not necessarily the closed orbit). DK denote the spin-flip transition probabilities per unit time from $\pm \hat{\mathbf{n}}$ to $\mp \hat{\mathbf{n}}$ by $p_{\downarrow}$ and $p_{\uparrow}$, respectively, where

$$
p_{\uparrow}=\frac{1}{4}\left\langle\int d \tau\left\langle 0\left|\omega \cdot \boldsymbol{\eta}_{t+\tau / 2} \omega \cdot \boldsymbol{\eta}_{t-\tau / 2}^{*}\right| 0\right\rangle\right\rangle,
$$

and similarly for $p_{\downarrow}$. The large angular brackets denote an average over orbits and $|0\rangle$ is the unperturbed initial state. BL absorb both of these into their angular brackets $\left\langle\delta \omega_{ \pm} \delta \omega_{\mp}\right\rangle$. It is easy to compare Eq. (45) with Eq. (43). First, transforming to the comoving frame, and restricting attention to the closed orbit,

$$
\boldsymbol{\eta}(\tau)=\boldsymbol{\eta}_{1}+i \boldsymbol{\eta}_{2}=(\hat{\mathbf{x}}+i \hat{\mathbf{y}}) \exp \left(-i \omega_{1} \tau\right) .
$$

Then, putting $t=0$ in Eq. (45),

$$
\boldsymbol{\omega} \cdot \boldsymbol{\eta}(\tau / 2)=\boldsymbol{\omega} \cdot(\hat{\mathbf{x}}+i \hat{\mathbf{y}}) \exp \left(-i \omega_{1} \tau / 2\right)
$$

and

$$
\boldsymbol{\omega} \cdot \boldsymbol{\eta}^{*}(-\tau / 2)=\boldsymbol{\omega} \cdot(\widehat{\mathbf{x}}-i \widehat{\mathbf{y}}) \exp \left(-i \omega_{1} \tau / 2\right)
$$

Putting $\omega_{ \pm}=\omega \cdot(\hat{\mathbf{x}} \pm i \hat{\mathbf{y}})$ and using BL angular brackets,

$p_{\uparrow, \downarrow}=\frac{1}{4} \int d \tau \exp \left(\mp i \omega_{1} \tau\right)\left\langle\omega_{ \pm}(\tau / 2) \omega_{\mp}(-\tau / 2)\right\rangle$,

and with small further changes of notation, this establishes the equivalence of the BL and DK expressions, except for the disagreement in Eq. (40). One should really be more precise in Eq. (46), because $p_{\uparrow, \downarrow}$ there are in the comoving frame, and are a factor $\gamma$ larger than their values in Eq. (45) (accelerator frame). Similarly, $\omega_{+}$and $\omega_{\text {- }}$ are not the same as in Eq. (45). This is not surprising, because of the time-dilation factor $\gamma$ relating the polarization buildup rates in these two frames. However, the equilibrium degree of polarization is the same, because

$$
P_{\mathrm{eq}}=\frac{p_{\uparrow}-p_{\downarrow}}{p_{\uparrow}+p_{\downarrow}}=\frac{\Gamma_{+}-\Gamma_{-}}{\Gamma_{+}+\Gamma_{-}} .
$$

Time-dilation factors cancel out between numerator and denominator in the above expressions.

Both sets of authors then derive their respective expressions for the equilibrium degree of polarization. The algebra involved need not concern us.

\section{CONCLUSIONS}

The main points of this paper have now been made, and remaining details can be left as an exercise for the reader. Some concluding remarks now follow. It has been shown that both Derbenev and Kondratenko and Bell and Leinaas have included the kinetic and statistical-mechanical subtleties of radiative electron polarization into their calculations, even though their formulations differ so much. The main point of attention has been the effect of vertical electron recoils on the polarization. Using the Derbenev-Kondratenko approach, the results of Refs. 1-3 were unified in Eqs. (41) and (42). However, numerically, the change is likely to be negligible, in comparison to the Derbenev-Kondratenko formula of Ref. 1. It so happens that $\gamma(\partial \widehat{\mathbf{n}} / \partial \gamma)$ vanishes in the quantitative calculations displayed in this paper, but it is an important vector in general. For the sake of completeness, and to offer the reader some feeling for what happens in models not considered in this paper, a 
few parenthetical statements on the behavior of $\gamma(\partial \hat{\mathbf{n}} / \partial \gamma)$ follow. In general,

$$
\left|\gamma \frac{\partial \hat{\mathbf{n}}}{\partial \gamma}\right| \gg \frac{2}{\gamma}\left|\frac{\partial \hat{\mathbf{n}}}{\partial \beta_{z}}\right|=f .
$$

It was already noted by Bell and Leinaas that with only one resonance, $f \rightarrow 0$ at high energies. In a model with several resonances the nonresonant part of $f$ remains approximately constant as the energy increases. However, the nonresonant part of $\gamma(\partial \widehat{\mathbf{n}} / \partial \gamma)$ actually increases with energy. Thus the increase in $P_{\text {eq }}$ seen by Bell and Leinaas near a resonance is not likely to be important at high energies.

The unified formula, Eq. (42), can be used in a modified version of the computer program SLIM of $\mathrm{Chao}^{8}$ or SMILE of Mane. ${ }^{4}$ Since for strong-focusing machines, the vertical $\beta$ function and the orbit curvature are no longer constant, the "new" derivative $\partial \widehat{\mathbf{n}} / \partial \beta_{b}$ will yield other first-order resonances corresponding to nonzero values of $k_{0}$ in Eq. (18), as well as higher-order resonances. However, Eq. (18) itself, which gives the spectrum of resonances, is not affected. In this connection it is interesting to note that in an earlier account by Chao of the SLIM formalism, ${ }^{9}$ in which he considered the effect of the vertical recoils, he would also have obtained Eq. (4.13) of Ref. 2, with $f$ given by Eq. (40). One can also show that the formalism of Ref. 9 yields the vertical emittance $\left\langle z^{2}\right\rangle$ obtained by Bell and Leinaas.

\section{ACKNOWLEDGMENTS}

One of us (D.P.B.) wishes to thank Professor L. N. Hand, Dr. H. Mais, and Dr. G. Ripken for helpful discussions. The other author (S.R.M.) would like to thank Dr. J. S. Bell for helpful communications and to acknowledge partial support from the U.S. Department of Energy under Contract No. DE-AC02-76ER01112.

\section{APPENDIX}

In this appendix some details of the partial derivatives of the DK spin-quantization axis $\hat{\mathbf{n}}$ with respect to $\gamma$ or $\beta_{z}$ will be given. The approach will follow that of Chao. ${ }^{8}$ The orbital trajectory $X(\theta)$ is written as a sixcomponent column vector $\left(x, x^{\prime}, z, z^{\prime}, \delta s, \Delta E / E_{0}\right)^{T}$, where $X=0$ is the closed orbit. Primes denote differentiation with respect to arc length. Then $X$ can be expressed as a sum of normal modes $E_{\lambda}$,

$$
X(\theta)=\sum_{\lambda= \pm 1, \pm 2, \pm 3} a_{\lambda} E_{\lambda},
$$

where the $a_{\lambda}$ are constants for a given trajectory. The notation is $E_{-\lambda}=E_{\lambda}^{*}$ and $a_{-\lambda}=a_{\lambda}^{*}$. Then the derivative $\gamma \partial X / \partial \gamma$ is given by considering two trajectories which differ by unity only in the sixth component, i.e.,

$$
\gamma \frac{\partial X}{\partial \gamma}=\sum_{\lambda} \gamma \frac{\partial a_{\lambda}}{\partial \gamma} E_{\lambda}=\left(\begin{array}{c}
0 \\
\vdots \\
0 \\
1
\end{array}\right)
$$

The $E_{\lambda}$ are normalized so that $E_{\lambda}^{\dagger} S E_{\lambda}=i$ for $\lambda>0$ and $E_{\lambda}^{\dagger} S E_{\lambda}=-i$ for $\lambda<0$, where

$$
S=\left(\begin{array}{cccccc}
0 & 1 & & & & \\
-1 & 0 & & & & \\
& & 0 & 1 & & \\
& & -1 & 0 & & \\
& & & & 0 & 1 \\
& & & & -1 & 0
\end{array}\right) \text {. }
$$

Blank spaces denote zeros in the above matrix. It then follows that

$$
\left.\left.\gamma \frac{\partial a_{\lambda}}{\partial \gamma}\right|_{\theta} \simeq \gamma_{0} \frac{\partial a_{\lambda}}{\partial \gamma}\right|_{\theta}= \begin{cases}-i E_{5 \lambda}^{*}(\theta), & \lambda>0 \\ i E_{5 \lambda}^{*}(\theta), & \lambda<0,\end{cases}
$$

where $E_{5 \lambda}$ is the fifth component of $E_{\lambda}$. Thus the partial differentiation $\gamma \partial / \partial \gamma$ can be effected by a simple substitution. By entirely similar reasoning, the derivative $\partial / \partial \beta_{z}$ can be obtained by demanding

$$
\frac{\partial X}{\partial z^{\prime}}=\sum_{\lambda} \frac{\partial a_{\lambda}}{\partial z^{\prime}} E_{\lambda}=\left(\begin{array}{l}
0 \\
0 \\
0 \\
1 \\
0 \\
0
\end{array}\right),
$$

and the result is

$$
\left.\frac{\partial a_{\lambda}}{\partial z^{\prime}}\right|_{\theta}= \begin{cases}-i E_{3 \lambda}^{*}(\theta), & \lambda>0 \\ i E_{3 \lambda}^{*}(\theta), & \lambda<0\end{cases}
$$

where $E_{3 \lambda}$ is the third component of $E_{\lambda}$. For a perfectly aligned planar machine, including the weak-focusing model considered here, $E_{5 \lambda}=0$ for the vertical (betatron) normal mode since there is no coupling between vertical and longitudinal oscillations. Furthermore, $E_{3 \lambda}$ for the vertical mode is a function only of the vertical $\beta$ function and phase. Specifically, for the weak-focusing model of BL,

$$
E_{3 \lambda}=\sqrt{\beta / 2} \exp \left(i Q_{z} \theta\right) \quad(\lambda>0) .
$$

There is one important point to note before using the above expressions in the main body of the text. It has to do with the coordinate system employed. Following standard accelerator physics practice, in this appendix the coordinate system is $\{x, z, s\}$, a right-handed coordinate system where $\hat{\mathbf{x}}$ is radial, $\widehat{\mathbf{z}}$ is vertical, and $\widehat{\mathbf{s}}$ is longitudinal. In the main body of the text, the BL coordinate system has been used, to simplify comparison with their work, and it is $\{x, y, z\}$, also a right-handed coordinate system, and where $\hat{\mathbf{x}}$ is still radial, but the second vector $\hat{\mathbf{y}}$ is longitudinal and $\hat{\mathbf{z}}$ is vertical. This implies a relative minus-sign difference between the coordinate systems for one of the unit vectors. Because of this, the correct expression for $\partial a_{z} / \partial \beta_{z}$ in Eq. (38) is $i \sqrt{\beta / 2} \exp \left(-i Q_{z} \theta\right)$, i.e., the above solution must be multiplied by a minus sign. 
${ }^{1}$ Ya. S. Derbenev and A. M. Kondratenko, Zh. Eksp. Teor. Fiz. 64, 1918 (1973) [Sov. Phys._JETP 37, 968 (1973)].

${ }^{2}$ Ya. S. Derbenev and A. M. Kondratenko, Zh. Eksp. Teor. Fiz. 62, 430 (1972) [Sov. Phys._JETP 35, 230 (1972)].

${ }^{3}$ J. S. Bell and J. M. Leinaas, Nucl. Phys. B 284, 488 (1987).

${ }^{4}$ S. R. Mane, Phys. Rev. A 36, 105 (1987); Ph.D. thesis, Cornell University, 1987 (unpublished).

${ }^{5} \mathrm{~K}$. Yokoya, Deutsches Elektronen-Synchroton (DESY) Report No. 86-057, 1986 (unpublished).
${ }^{6}$ A. A. Sokolov and I. M. Ternov, Dokl. Akad. Nauk. SSSR 153, 1052 (1963) [Sov. Phys._Dokl. 8, 1203 (1964)].

${ }^{7} \mathrm{~K}$. Yokoya, National Laboratory for High Energy Physics (KEK) Report No. 81-19, 1982 (unpublished); S. R. Mane, DESY Report No. 85-125, 1985 (unpublished).

${ }^{8}$ A. W. Chao, Nucl. Instrum. Meth. 29, 180 (1981).

${ }^{9}$ A. W. Chao, Stanford Linear Accelerator Center, Stanford University Report No. SPEAR-208/PEP-257, 1977 (unpublished); J. Appl. Phys. 50, 595 (1979). 